\title{
An Approach for Canny Edge Detection Algorithm on Face Recognition
}

\author{
A. D. Chitra ${ }^{1}$, Dr. P. Ponmuthuramalingam ${ }^{2}$ \\ ${ }^{1}$ Assistant Professor, Department of Computer Science, Coimbatore Institute Of Technology, Coimbatore - 641014 \\ ${ }^{2}$ Associate Professor and Head, PG \& Research, Department of Computer Science, Government Arts College(Autonomous), Coimbatore- \\ 641018
}

\begin{abstract}
The paper obtainable here is a challenge to use a canny edge detection algorithm for face recognition. Defined an edge as a set of related pixels that forms a margin between two disjoints regions, the automatic recognition of human faces grants an important challenge to the research community of pattern recognition. There are differences in the lighting, angles, facial expressions and poses, further set a problem to the face recognition research. This paper proposes a new approach, canny edge detection method for face recognition and image color space transformation, Gaussian Filter Coefficient and Hysteresis thresholding using two values for thresholding, High and Low. The proposed method works efficiently with images and performs better than all other edge detection algorithm.
\end{abstract}

Keyword: Canny edge detection algorithm, Gaussian Filter, Hysteresis thresholding

\section{Introduction}

Facial recognition is a visual pattern recognition task. The three-dimensional human face, which is subject to varying illumination, pose, expression etc. has to be recognized. This recognition can be performed on a variety of input data sources. The aim of face detection is localization of the face in a image. In the case of video input, it can be an advantage to track the face in between multiple frames, to reduce computational time and preserve the identity of a face (person) between frames. The face preprocessing step is to normalize the coarse face detection, so that a robust feature extraction can be achieved. Depending of the application, face preprocessing includes: Alignment (translation, rotation, scaling) and light normalization/correlation. The dimensionality of these sources can be increased by one by the inclusion of a time dimension. A still image with a time dimension is a video sequence. The advantage is that the identification of a person can be determined more precisely from a video sequence than from a picture since the identity of a person cannot change from two frames taken in sequence from a video sequence. Facial recognition systems usually consist of four steps, as face detection (localization), face preprocessing face alignment/normalization, light correction and etc.), feature extraction and feature matching.

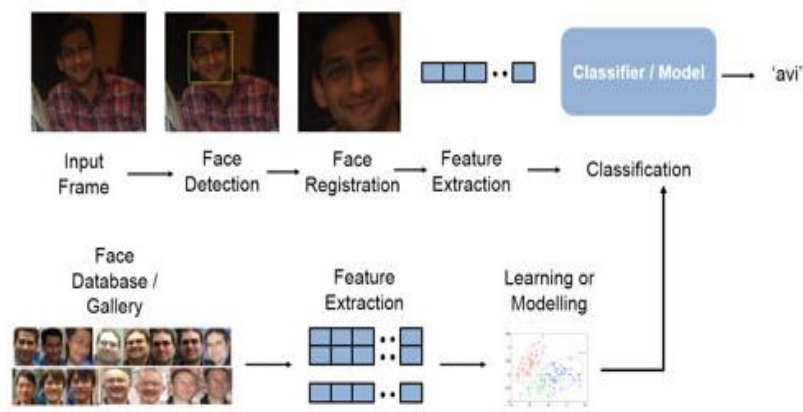

Figure 1: Facial recognition process

\section{Literature Survey}

Zhi-fang et al. [16] Although face recognition systems are known for decades, there are many active research work on the topic. The subject can be divided into three parts;

1. Detection

2. Recognition

The first step is face detection for face recognition system. The detection output can be location of face region as a whole, and location of face region through facial types (i.e. eyebrow, nose, eyes, mouth etc.). Detection methods in the works are difficult to classify strictly, because most of the algorithms are combination of methods for detecting faces to increase the accuracy. Kherchaoui and Houacine modeled skin color using Gaussian Distribution Model with $\mathrm{Cb}$ and $\mathrm{Cr}$ channel in $\mathrm{YCbCr}$ color space. Then skin like region is chosen as a face candidate with respect to the bounding box ratio of the region and candidates are verified with template matching. Another method preprocesses the given image to remove background part as a first step. It is done by applying edge detection on the $\mathrm{Y}$ component of $\mathrm{YCbCr}$ color space. Then, the closedregion is filled to take it as foreground part. After that, skin segmentation is done on $\mathrm{YCrCb}$ color space with conditions. The segmented parts are taken as candidate and verification is done by calculating the entropy of the candidate image and use thresholding to verify face. Face recognition techniques are described in previous paragraphs. Selection of thebest algorithm cannot be done because of nonstandard face libraries and nonuniversal face databases. Also, detection and recognition method can be affected by illumination changes, pose changes, occlusion, and facial expression. All methods cannot handle all effects in the same algorithm. So, it is difficult to design an algorithm that handles all effects.

\section{Canny Edge Detection Algorithm}

Canny's edge detector is one of the most popular edge detection algorithms. Canny proposed a list of criteria to improve edge detection method. 


\section{International Journal of Science and Research (IJSR) \\ ISSN (Online): 2319-7064}

Index Copernicus Value (2013): 6.14 | Impact Factor (2014): 5.611

Algorithm Steps:

The first criterion is low error rate. That is genuine edges should not be missed and spurious edges not picked.

The second criterion is good localization. In other words, the pixels edges are found between distance by the detector and the actual edge should be minimal.

A third criterion is single edge point have only one response. This was cited since the first two are not considerable enough to totally eliminate multiple responses to an edgefor possibility. Established on these criteria, the canny's edge detector was designed.

The first step in the process is smoothing the image using a Gaussian filter. Then the image Gradient is computed using multiple first derivative operators in different directions, to highlight regions with high spatial derivatives. Then the algorithm tracks along these regions not at the maximum with the pixel that is non-maximum suppression. The array gradient is then further refined by Hysteresis. Track along the remaining pixels using hysteresis is not been suppressed. That is, first two thresholds (lower T1 and upper T2) are identified. Then points whose magnitudes are below the lower threshold are set to zero (i.e. made a non edge). Points whose magnitudes are above the upper threshold is made an edge. Those points whose magnitude is between the 2 thresholds, is fixed to zero except there is a track from this point to a point with a gradient above T2. The performance of the Canny's algorithm depends on number of parameters such as $\sigma$, standard deviation for the Gaussian filter and lower and upper threshold values $\mathrm{T} 1$ and $\mathrm{T} 2$. The value of $\sigma$ controls the size of the Gaussian filter. Larger the scale of the Gaussian filter, lesser the accuracy of localization of the edges. Whereas smaller values of $\sigma$ imply a smaller Gaussian filter which limits the amount of blurring, maintaining finer edges in the image. As can be seen, Canny's algorithm performs better than all the other gradient based methods and LoG filters in detecting all the fine edges and localizing them correctly. However the drawback of the Canny's algorithm is it generates lots of spurious edges corresponding to weak edge points in the image.

\section{Experimental Results}

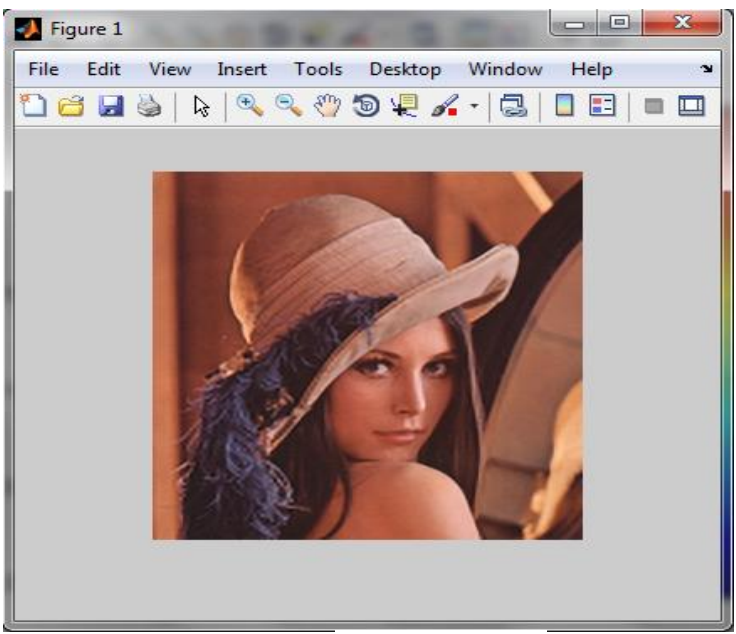

Figure 1: Gaussian Filter

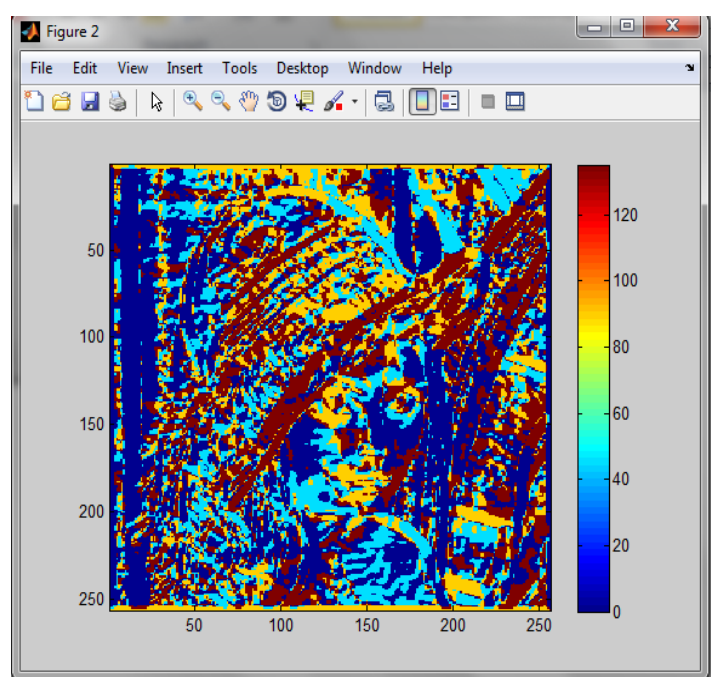

Figure 2: Filter for horizontal and vertical orientation

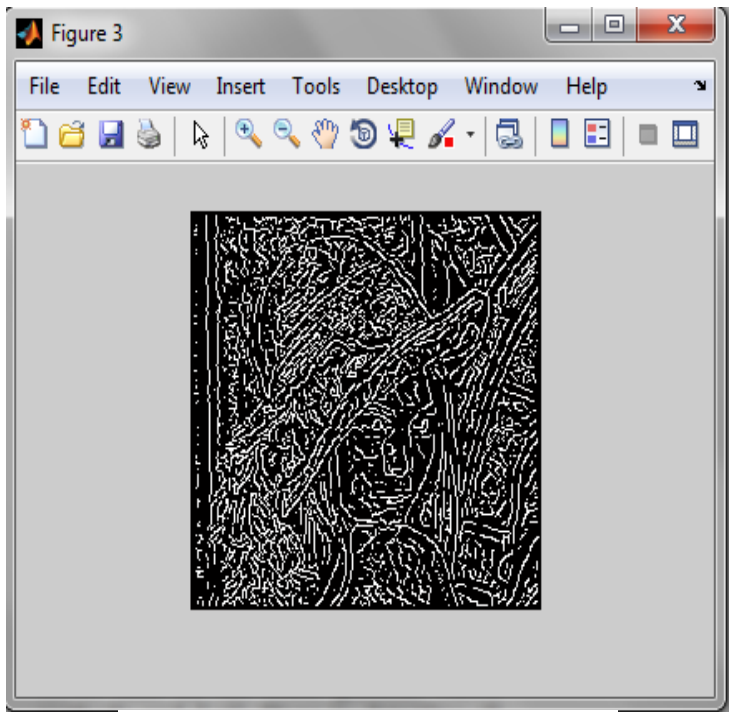

Figure 3: Hysteresis Threshold low

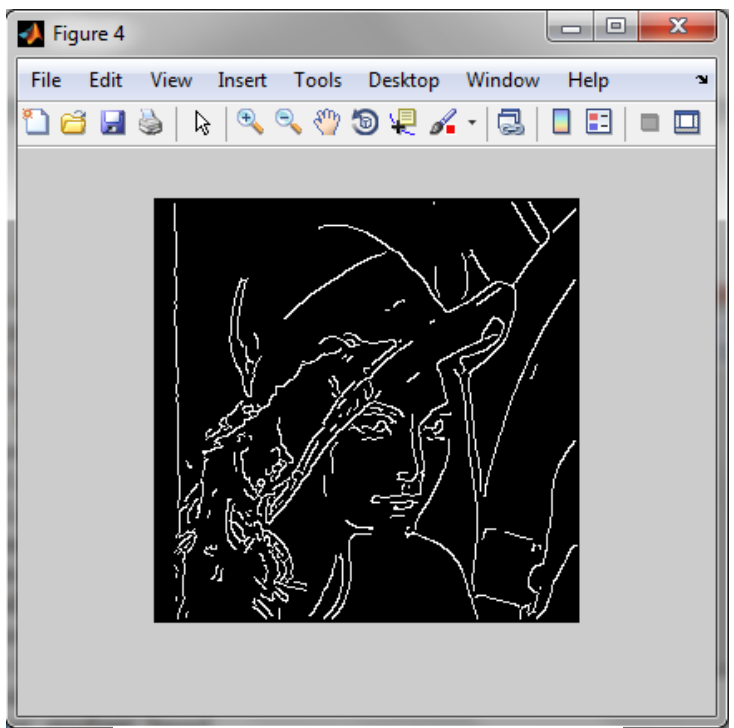

Figure 4: Hysteresis Threshold high 


\section{International Journal of Science and Research (IJSR) \\ ISSN (Online): 2319-7064}

Index Copernicus Value (2013): 6.14 | Impact Factor (2014): 5.611

Table 1: Parameter Setting and time

\begin{tabular}{|lcc|}
\hline Algorithm & Image 1 & Image 2 \\
\hline Canny Algorithm & $\sigma=2$ & $\sigma=2$ \\
Parameters & $\begin{array}{r}\text { Adaptive } \\
\text { Threshold }\end{array}$ & $\begin{array}{c}\text { Adaptive } \\
\text { Threshold }\end{array}$ \\
Time & $41 \mathrm{~ms}$ & $51 \mathrm{~ms}$ \\
\hline
\end{tabular}

We select an image file and apply canny algorithm with the following steps and the result is shown in figures

1) Convolution with Gaussian Filter Coefficient

2) Canny Filter for Horizontal and Vertical orientation with convolution.

3) Directions calculated using a tan

4) Adjusting to nearest $0,45,90$, and 135 degree.

5) Non-Maximum Suppression

6) Hystheresis Thresholding (high and low)

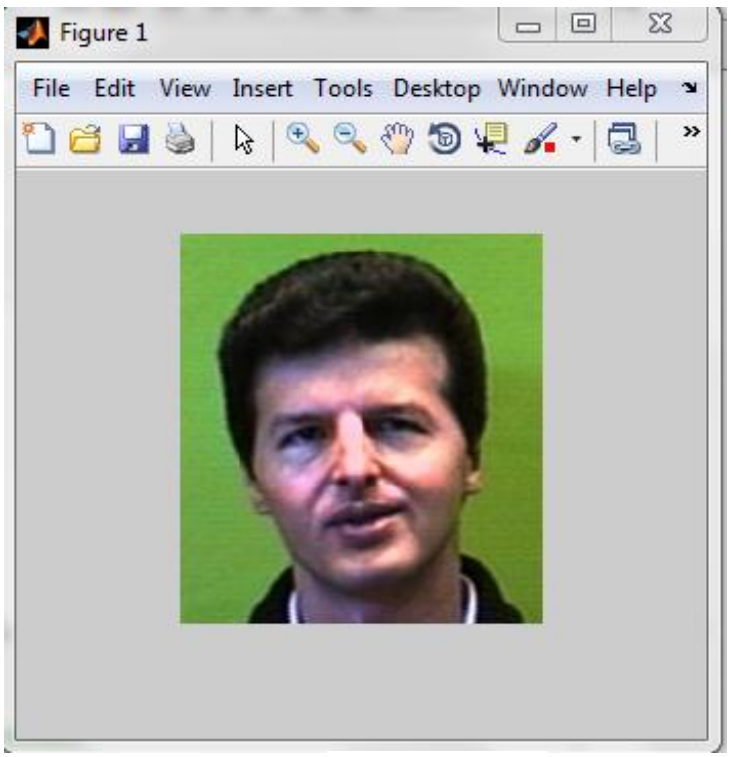

Figure 1: Gaussian Filter

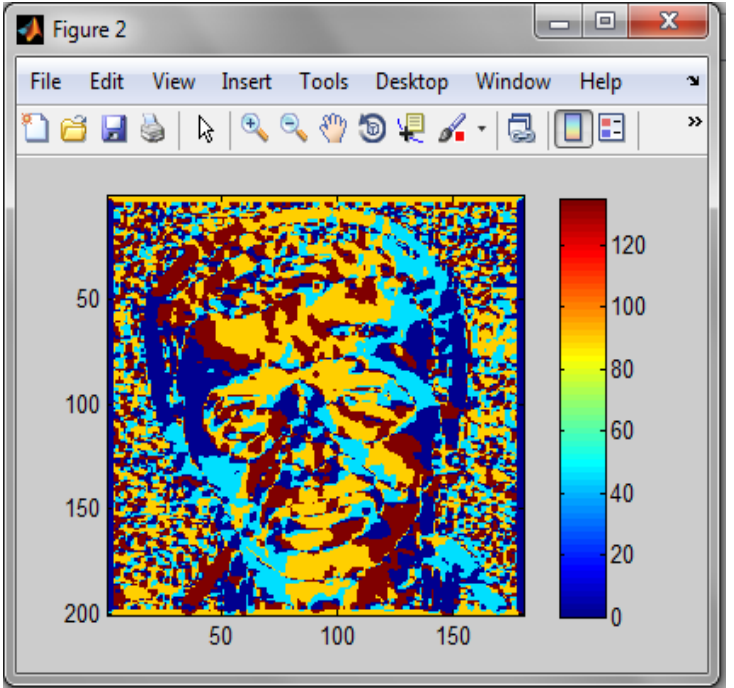

Figure 2: Filter for horizontal and vertical orientation

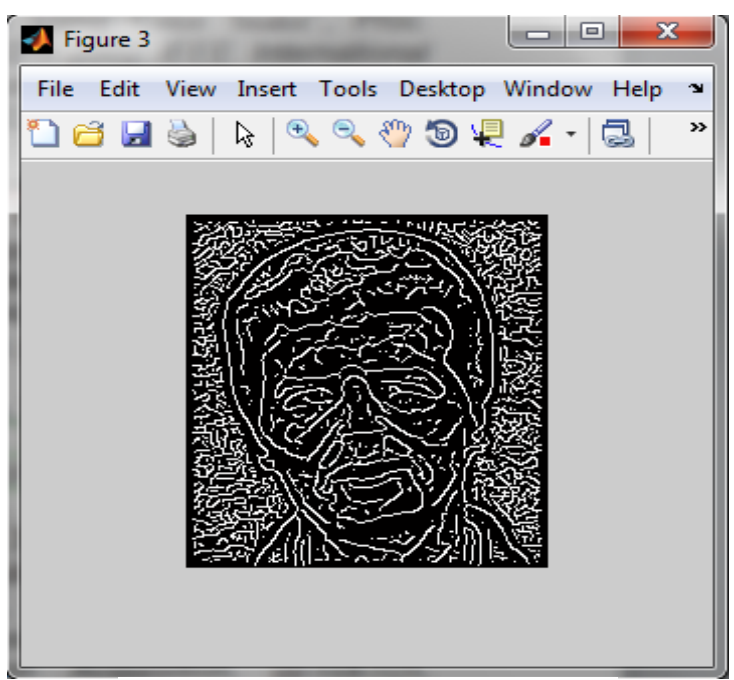

Figure 3: Hysteresis Threshold low

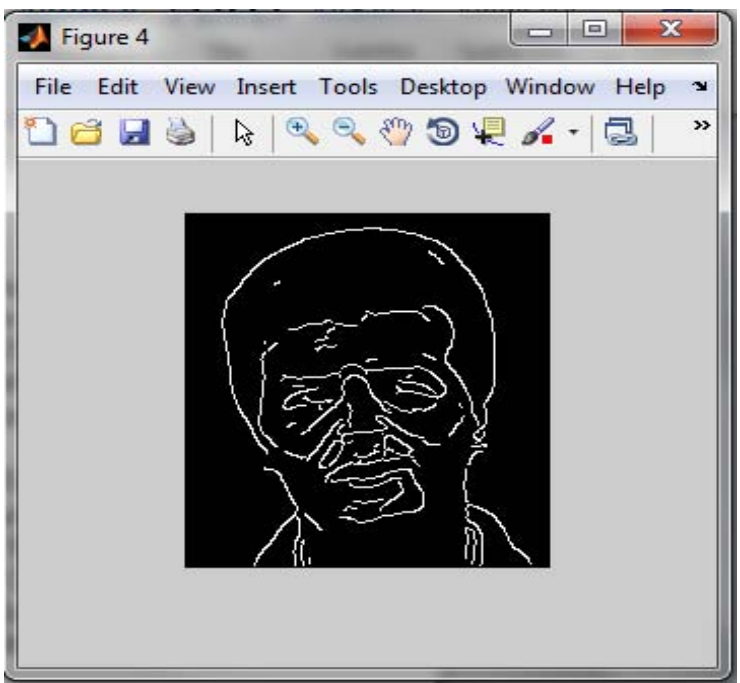

Figure 4: Hysteresis Threshold high

\section{Conclusion}

Canny operator can be applied to different situations. Canny operator can detect the edge clearly. In order to improve its performance, we propose the calculation of gradient magnitude and gradient direction based and make the adaptive calculation of the threshold of Canny operator method. The experimental results show that the edge detected by the improved Canny operator has more continuity, and greater to noise ratio.

\section{References}

[1] AdityaKelkar, " Face Recognition using Eigenfaces Approach".

[2] H.S.Bhadauria1, Annapurna Singh2, Anuj Kumar3 .2013 "Comparison between Various Edge Detection Methods on Satellite Image" International Journal of Emerging Technology and Advanced Engineering Website: www.ijetae.com (ISSN 2250-2459, ISO 9001:2008 Certified Journal, Volume 3, Issue 6, 324

[3] Caifeng Shan, Shaogang Gong W. McOwan., 2009, "Facial expression recognition based on Local Binary Pattrens: A Comparartive Study “ , Image and Vision Computing 27,803-816. 
[4] R.Chellapa, L. Wilson and S.Sironey 1995 "Human and machine Recognition of faces: A Survey “,Proc IEEE, vol 83,pp 705-740,.

[5] DimitriPissarenko, “ Eigenface -based facial recognition".

[6] Jolly D. shah and S.H.Patil "Biometric Authentication based on Detection and Recognition of Multiple faces in Image " UFL\& JIITU, IC3-208, pp87-96.

[7] N.krishnan, GR jothi, K Pullor, GL Aaron "Recognition of Non-symmetric faces using principal Component Analysis.

[8] Ming-Hsuan Yang, "Recent Advances in Face Recognition".

[9] Ping ZHOU1,Wenjun YE1, Yaojie XIA1, Qi WANG2., May 2011 "An Improved Canny Algorithm for Edge Detection" Journal of Computational Information Systems 7:5 (1516-1523)

[10] Rabiajafri and Hamid R.Arabnia , 2009. "A Survey of face Recognition Technique," Jour . of Information Processing Systems, vol.5, June

[11] Sheifali Gupta, O.P.Sahoo, Ajay Goel, Rupesh Gupta. 2010"A new Optimized Approach to Face Recognition Using Eigen Faces". JCST.

[12] Shokhan M. H., 2014 "An efficient approach for improving Canny Edge Detection Algorithm " International Journal of Advances in Engineering \& Technology,. CIJAET ISSN: 2231196359 Vol. 7,Issue 1, pp. 59-65

[13] G.T. Shrivakshan, Dr. C. Chandrasekar., 2012 "A Comparison of various Edge Detection Techniques used in Image Processing" IJCSI International Journal of Computer Science Issues, Vol. 9, Issue 5, No 1, ISSN (Online): 1694-0814

[14] M.Turk and A.Pentland, 1991 "Face Recognition using Eigenfaces." Prodeedings ,IEEE Conference on Computer Vision and Pattern Recognition, pages 586591,.

[15] Y.VijayaLata, Dr.A.Govardhan, Dr.L.P. Reddy., 2009 "Facial recognition using Eigenfaces by PCA" .IJRTIE,

[16] VinayHiremath, AswiniMayakar "Face recognition using Eigenface Approach"

[17] YogeshTayal, PramodKUmarPandey, D.B.V.Singh., 2013 ."Face recognition using eigenfaces. IJETCAS ,.

[18] W.Zhao. R .Chellappa, P.J. Phillips and A. Rosenfeld, "Face Recognition : A literature survey"

[19] Wikipedia.http://en.wikipedia.org/wiki/Eigenvalue, ei genvctor_and_eigenspace[19] L. Zhi-fang, Y. Zhisheng, A.K.Jain and W. Yun-qiong, 2003, "Face Detection And Facial Feature Extraction In Color Image", Proc. The Fifth International Conference on Computational Intelligence and Multimedia Applications(ICCIMA'03), pp.126-130, Xi'an, China.

[20] S. Kherchaoui and A. Houacine, 2010, "Face Detection Based On A Model Of The Skin Color With Constraints And Template Matching", Proc. 2010International Conference on Machine and Web Intelligence, pp. 469 - 472, Algiers. 